\title{
Bilayer graphene with single and multiple electrostatic barriers: Band structure and transmission
}

\author{
Michaël Barbier, ${ }^{1 *}$ P. Vasilopoulos, ${ }^{2, \dagger}$ F. M. Peeters, ${ }^{1, \ddagger}$ and J. Milton Pereira, Jr. ${ }^{3, \S}$ \\ ${ }^{1}$ Department of Physics, University of Antwerp, Groenenborgerlaan 171, B-2020 Antwerpen, Belgium \\ ${ }^{2}$ Department of Physics, Concordia University, 7141 Sherbrooke Ouest, Montréal, Quebec, Canada H4B 1R6 \\ ${ }^{3}$ Departamento de Física, Universidade Federal do Ceará, Fortaleza, Ceará 60455-760, Brazil \\ (Received 11 September 2008; revised manuscript received 2 March 2009; published 1 April 2009)
}

\begin{abstract}
We evaluate the electronic transmission and conductance in bilayer graphene through a finite number of potential barriers. Further, we evaluate the dispersion relation in a bilayer graphene superlattice with a periodic potential applied to both layers. As a model we use the tight-binding Hamiltonian in the continuum approximation. For zero bias the dispersion relation shows a finite gap for carriers with zero momentum in the direction parallel to the barriers. This is in contrast to single-layer graphene where no such gap was found. A gap also appears for a finite bias. Numerical results for the energy spectrum, conductance, and the density of states are presented and contrasted with those pertaining to single-layer graphene.
\end{abstract}

DOI: 10.1103/PhysRevB.79.155402

PACS number(s): 71.10.Pm, 73.21.-b, 81.05.Uw

\section{INTRODUCTION}

Low-dimensional systems have long been the subject of intensive research both on their fundamental properties and on possible applications. In this respect the recent production of atom-thick crystal carbon layers (graphene) has raised the possibility of the development of new graphene-based devices that exploit its unusual electronic and mechanical properties (for a recent review see Ref. 1). The electronic spectrum of defect-free single-layer graphene is gapless and, together with the chiral aspect of the carriers in this system, leads to a perfect transmission through an arbitrarily high and wide potential barrier, i.e., the Klein paradox. ${ }^{2,3}$ That can be avoided if a gap is introduced in the electronic spectrum and may be necessary for certain applications, e.g., for improving the on/off ratio in carbon-based transistors.

There are a few methods to introduce a gap in the spectrum of graphene. One of them is to use nanoribbons in which a band gap ${ }^{4}$ arises due to the lateral confinement. Also, depositing graphene on a substrate such as boron nitride was found recently to result in a band gap ${ }^{5}$ of $53 \mathrm{meV}$. In bilayer graphene ${ }^{6}$ a gap can be introduced by applying a bias between the two layers or by doping one of them such that a potential difference results between the layers. ${ }^{7-10}$ Changing the bias in the latter case can open and close the gap dynamically which is interesting for transistor applications. Nanostructured gates can thus allow the creation of quantum dots on bilayer graphene. ${ }^{11}$

In this paper we investigate the electronic properties of a biased bilayer in which the potential difference between the two layers is changed periodically. Such a superlattice (SL), which can be created by applying gates to the bilayer, is of interest as it shows how a one-dimensional (1D) band structure may appear in such a system. An additional motivation is that curvature effects of corrugated single-layer graphene lead to an effective periodic potential resembling that of a SL. ${ }^{12}$ Although in a bilayer this effect would be weaker, since the bilayer is less bendable than a single layer, it might still be important.

This paper is organized as follows. Section II briefly shows the basic formalism. In Sec. III results for the trans- mission and conductance through a finite number of barriers are presented. Section IV shows results for the dispersion relation and the density of states (DOS) in SLs in bilayer graphene. Finally, a summary and concluding remarks are given in Sec. V.

\section{HAMILTONIAN, ENERGY SPECTRUM, AND EIGENSTATES}

Bilayer graphene consists of two $A-B$-stacked monolayers of graphene. Each monolayer has two independent atoms $A$ and $B$ in its unit cell. The relevant Hamiltonian, obtained by a nearest-neighbor tight-binding approximation near the $K$ point, and the eigenstates $\Psi$ read

$$
\mathcal{H}=\left(\begin{array}{cccc}
V_{1} & \pi & t_{\perp} & 0 \\
\pi^{\dagger} & V_{1} & 0 & 0 \\
t_{\perp} & 0 & V_{2} & \pi^{\dagger} \\
0 & 0 & \pi & V_{2}
\end{array}\right), \quad \psi=\left(\begin{array}{c}
\psi_{A} \\
\psi_{B} \\
\psi_{B^{\prime}} \\
\psi_{A^{\prime}}
\end{array}\right) .
$$

Here $\pi=v_{F}\left(p_{x}+i p_{y}\right), p_{x, y}=-i \hbar \partial_{x, y}$ is the momentum operator, $v_{F}=10^{6} \mathrm{~m} / \mathrm{s}$ is the Fermi velocity, $V_{1}$ and $V_{2}$ are the potentials on layers 1 and 2 , respectively, and $t_{\perp}$ describes the coupling between these layers. As shown in the Appendix, for spatially independent $t_{\perp}, V_{1}$, and $V_{2}$, the spectrum consists of four bands given by

$$
\begin{aligned}
& \varepsilon_{ \pm}^{\prime+}=\left[\epsilon_{k t^{\prime}}^{2} \pm t^{\prime} \sqrt{4 k^{2} \delta^{2} / t^{\prime 2}+k^{2}+t^{\prime 2} / 4}\right]^{1 / 2}, \\
& \varepsilon_{ \pm}^{\prime-}=-\left[\epsilon_{k t^{\prime}}^{2} \pm t^{\prime} \sqrt{4 k^{2} \delta^{2} / t^{\prime 2}+k^{2}+t^{\prime 2} / 4}\right]^{1 / 2} .
\end{aligned}
$$

Here $\quad \epsilon_{k t^{\prime}}^{2}=k^{2}+\delta^{2}+t^{\prime 2} / 2, \quad \Delta=\left(V_{1}-V_{2}\right), \quad \delta=\Delta / 2 \hbar v_{F}, \quad \varepsilon$ $=E / \hbar v_{F}$, and $t^{\prime}=t_{\perp} / \hbar v_{F}$. The eigenstates $\psi$ of $\mathcal{H}$ are given by Eq. (A11) in the Appendix.

A reduced version of the four-band Hamiltonian shown in Eq. (1) that is often used ${ }^{9}$ is given by

$$
\mathcal{H}=-\frac{v_{F}^{2}}{t_{\perp}}\left(\begin{array}{cc}
0 & \pi^{\dagger^{2}} \\
\pi^{2} & 0
\end{array}\right)+V(x) \rrbracket,
$$

where 1 is the $2 \times 2$ unit matrix. Assuming solutions of the form $A \exp \left(i k_{x} x\right) \exp \left(i k_{y} y\right)$ we can replace $p_{x}$ by $\hbar k_{x}$. Then 
setting the determinant of the equation $\mathcal{H} \psi=E \psi$ equal to zero gives rise to the two-band spectrum

$$
E-V= \pm\left(v_{F}^{2} \hbar^{2} / t_{\perp}\right)\left(k_{x}^{2}+k_{y}^{2}\right),
$$

where $V$ is the potential applied to each layer. In Sec. III we compare some of the results obtained from Eqs. (1) and (2) with those obtained from Eqs. (3) and (4).

\section{FINITE NUMBER OF BARRIERS}

\section{A. Transmission}

When applying a bias to a metallic strip a potential barrier is created; we will approximate it by a square potential barrier. The eigenstates $\Psi$ given in the Appendix can be used in each region of constant potential. In matrix notation the wave function in region $j$ with a constant potential can be written as a matrix product [cf. Eq. (A13) of the Appendix],

$$
\Psi_{j}=\mathcal{G}_{j} \mathcal{M}_{j} \mathcal{A}_{j},
$$

where $\mathcal{A}_{j}=\left[A_{j}, B_{j}, C_{j}, D_{j}\right]^{T}$ and the superscript $T$ denotes the transpose of the row vector. Then we apply the continuity of the wave function at different potential steps. For the $(j$ $+1)$ th potential step at $x_{j+1}$ we obtain

$$
\mathcal{A}_{j+1}=\mathcal{M}_{j+1}^{-1}\left(x_{j+1}\right) \mathcal{G}_{j+1}^{-1} \mathcal{G}_{j} \mathcal{M}_{j}\left(x_{j+1}\right) \mathcal{A}_{j} .
$$

This links the coefficients of the wave function behind the barriers to those in front of them. Then we can write

$$
\mathcal{A}_{n+1}=\mathcal{N} \mathcal{A}_{1},
$$

where $\mathcal{N}_{j}=\mathcal{M}_{j+1}^{-1}\left(x_{j+1}\right) \mathcal{G}_{j+1}^{-1} \mathcal{G}_{j} \mathcal{M}_{j}\left(x_{j+1}\right)$ and $\mathcal{N}=\prod_{j} \mathcal{N}_{j}$. From now on we assume $|E|<t_{\perp}$ outside the barrier such that $\alpha_{+}$ $\in \mathbb{R}$ and $\alpha_{-} \in \mathrm{C}$, see the Appendix. Assuming that there is an incident wave, with wave vector $\alpha_{+}$from the left (normalized to unity), part of it will be reflected (coefficient $r$ ) and part of it will be transmitted (coefficient $t$ ). Also there are growing and decaying evanescent states near the barrier (coefficients $e_{g}$ and $e_{d}$, respectively). The relation between all these waves is written in the form

$$
\mathcal{N}\left(\begin{array}{c}
t \\
0 \\
e_{d} \\
0
\end{array}\right)=\left(\begin{array}{c}
1 \\
r \\
0 \\
e_{g}
\end{array}\right)
$$

It can be rewritten as a linear system of equations,

$$
\left(\begin{array}{l}
1 \\
0 \\
0 \\
0
\end{array}\right)=\left(\begin{array}{cccc}
N_{11} & 0 & N_{13} & 0 \\
N_{21} & -1 & N_{23} & 0 \\
N_{31} & 0 & N_{33} & 0 \\
N_{41} & 0 & N_{43} & -1
\end{array}\right)\left(\begin{array}{c}
t \\
r \\
e_{d} \\
e_{g}
\end{array}\right),
$$

where $N_{i j}$ are the coefficients of $\mathcal{N}$. By inverting the square matrix in Eq. (9), which we label as $\mathcal{M}$, the coefficients can be calculated from $\left(\begin{array}{llll}t & e_{d} & e_{g}\end{array}\right)^{T}=\mathcal{M}^{-1}\left(\begin{array}{llll}1 & 0 & 0 & 0\end{array}\right)^{T}$. Thus, in order to obtain the transmission amplitude $t$ it is sufficient to find the matrix element $\left(\mathcal{M}^{-1}\right)_{11}$, which is given by $\left(\mathcal{M}^{-1}\right)_{11}=\left[N_{11}-N_{13} N_{31} / N_{33}\right]^{-1}$. For the general case the set
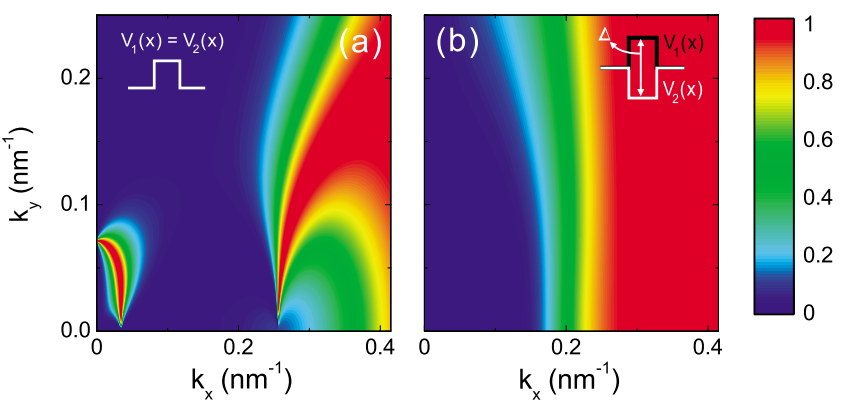

FIG. 1. (Color online) Transmission through a single square barrier of height $V=100 \mathrm{meV}$ and width $10 \mathrm{~nm}$. The potential difference $\delta=\left(V_{1}-V_{2}\right) / 2 \hbar v_{F}$ is zero in panel (a) and $\Delta=100 \mathrm{meV}$ in panel (b) inside the barrier/well region.

of equations above can be solved numerically and the transmission is given by $T=|t|^{2}$. If $k_{y}=0$ the system can be solved analytically for a single square barrier, of thickness $D$, without bias since the localized parts of the wave function are decoupled from the propagating ones. Then

$$
t=e^{i \alpha_{1} D}\left[\cos \left(\alpha_{2} D\right)-i Q \sin \left(\alpha_{2} D\right)\right]^{-1},
$$

where

$$
Q=\frac{1}{2}\left(\frac{\varepsilon_{1} \alpha_{2}}{\alpha_{1} \varepsilon_{2}}+\frac{\alpha_{1} \varepsilon_{2}}{\varepsilon_{1} \alpha_{2}}\right),
$$

with $\varepsilon_{j}=\left(E-V_{j}\right) / \hbar v_{F}$ in region $j$ and the momenta along the $x$ direction $\alpha_{1}$ and $\alpha_{2}$, outside and inside the barrier, respectively, given by $\alpha_{j}=\left[\varepsilon_{j}^{2}+\varepsilon_{j} t_{\perp}\right]^{1 / 2}$. The analog of Eq. (10) for the two-band Hamiltonian Eq. (3) is the same but with $\alpha_{j}$ $=\left[\varepsilon_{j} t_{\perp}\right]^{1 / 2}$ instead of $\alpha_{j}=\left[\varepsilon_{j}^{2}+\varepsilon_{j} t_{\perp}\right]^{1 / 2}$. This contrasts with the standard formula for the transmission probability obtained from the Schrödinger equation. For a system of two barriers at distance $L$ as in Fig. 2(a) we can obtain the transmission for $k_{y}=0$ analytically. The result is $T_{d}=\left|t_{d}\right|^{2}$ where

$$
t_{d}=\frac{e^{i 2 \alpha_{1}(L+2 D)}|t|^{2} e^{i 2 \phi_{t}}}{1-|r|^{2} e^{i 2 \phi_{r}} e^{i 2 \alpha_{1} L}},
$$

$r \equiv|r| e^{i \phi_{r}}$, and $t \equiv|t| e^{i \phi_{t}}$. Resonances will appear when $e^{i 2 \phi_{r}} e^{i 2 \alpha_{1} L}=1$, where $\phi_{r}$ is independent of the distance $L$. We obtain more resonances if $L$ increases.

A contour plot of the transmission through a single barrier is shown in Fig. 1. Panel (a) is for a barrier with height 100 $\mathrm{meV}$ and width $10 \mathrm{~nm}$ and the potential difference $\Delta$ between the layers is zero. In contrast to the case of a twodimensional electron gas (2DEG), there are transmission resonances for energies lower than the barrier height. These are due to hole states inside the barrier through which the electrons can tunnel. ${ }^{13}$ In panel (b) the barrier is $10 \mathrm{~nm}$ wide and the potential difference between the layers is $\Delta$ $=100 \mathrm{meV}$. As seen, the $k_{y}$ dependence of the transmission in panel (b) is weaker than that on $k_{x}$ and resembles the Schrödinger case. The electron transmission at normal incidence $\left(k_{y}=0\right)$ starts at $k_{x} \simeq 0.23 \mathrm{~nm}^{-1}$, corresponding to an energy of $50 \mathrm{meV}$ which is at the edge of the gap, inside which the states are evanescent and the transmission is suppressed. 


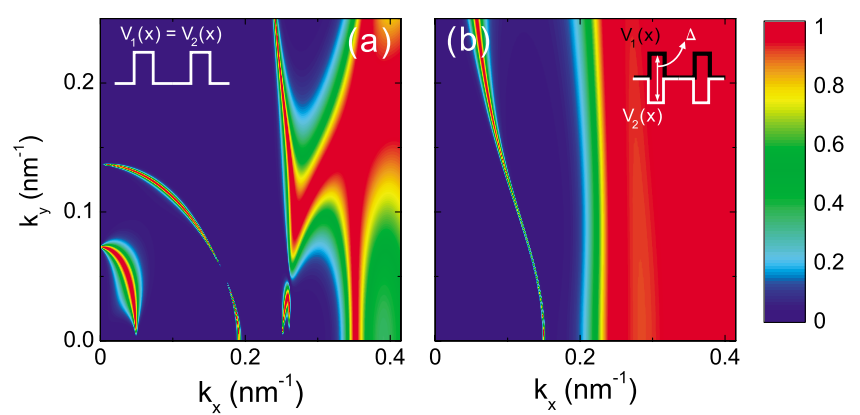

FIG. 2. (Color online) Transmission through a double barrier. The square barriers are $100 \mathrm{meV}$ high and $10 \mathrm{~nm}$ wide; the distance between them is $L=10 \mathrm{~nm}$. In panel (a) the potential difference between the layers is zero; in panel (b) it is $100 \mathrm{meV}$ inside the barrier/well regions.

Plots of transmission as function of wave vector through double barriers are shown in Fig. 2. The barriers are 100 meV high, $10 \mathrm{~nm}$ wide, the distance between the barriers is $L=10 \mathrm{~nm}$, and $\Delta=0$ for panel (a), whereas panel (b) shows results for $\Delta=100 \mathrm{meV}$. In agreement with Ref. 14, we find that it is the distance $L$ between the barriers and not their width that is important in determining the tunneling states and thus the transmission. For more results, e.g., conduction through unbiased multiple-barrier systems, see Refs. 14 and 15. For the double-barrier systems shown in Fig. 2, extra resonances are found and are associated with quasibound states in the well. It is seen from the derivation above for the one-dimensional case that the effect of these states increases with the interbarrier distance. In Fig. 2(b) a resonance occurs at $k_{x} \simeq 0.16 \mathrm{~nm}^{-1}$ and results from states available in the well between the barriers. In contrast to the single-layer case, in Figs. 1(a) and 2(a) there is no perfect transmission for normal incidence $\left(k_{y}=0\right)$, even though the system is gapless. This is a consequence of the chiral nature of the carriers in bilayer graphene, see, e.g., Ref. 3.

The transmission plots shown in Figs. 1 and 2 depend on the angle of incidence $\phi$ given by $\tan \phi=k_{y} / k_{x}$. A more direct way to see that is shown in Fig. 3 where the transmission is plotted as function of the angle of incidence for constant energy $E=17 \mathrm{meV}$. For panel (a) we used the $4 \times 4$ Hamiltonian of Eq. (1), with bias $\Delta=0$, while for panel (b) we used the $2 \times 2$ one of Eq. (3). The figure shows a significant discrepancy between the angular dependence as calculated from the two-band and the four-band Hamiltonians. This can be explained by the fact that the position of the resonances depends strongly on the wave-vector matching at each region; a slight change in the value of $\alpha_{j}$, for a given energy, may result in a large modification of the angular position of the transmission resonance.

The two-band parabolic approximation of Eqs. (3) and (4) can be expected to hold if both $\varepsilon_{1}$ and $\varepsilon_{2}$ are small compared to $t_{\perp}$, since in that regime the nonparabolicity of the spectra in all regions, as given by Eq. (2), can be neglected. That means that the two-band Hamiltonian can give accurate results only in the regime of low barrier heights and low incident electrons energies. Therefore, we consider the results of panel (a) to be more accurate than those of panel (b) for the energies and barrier heights considered here.
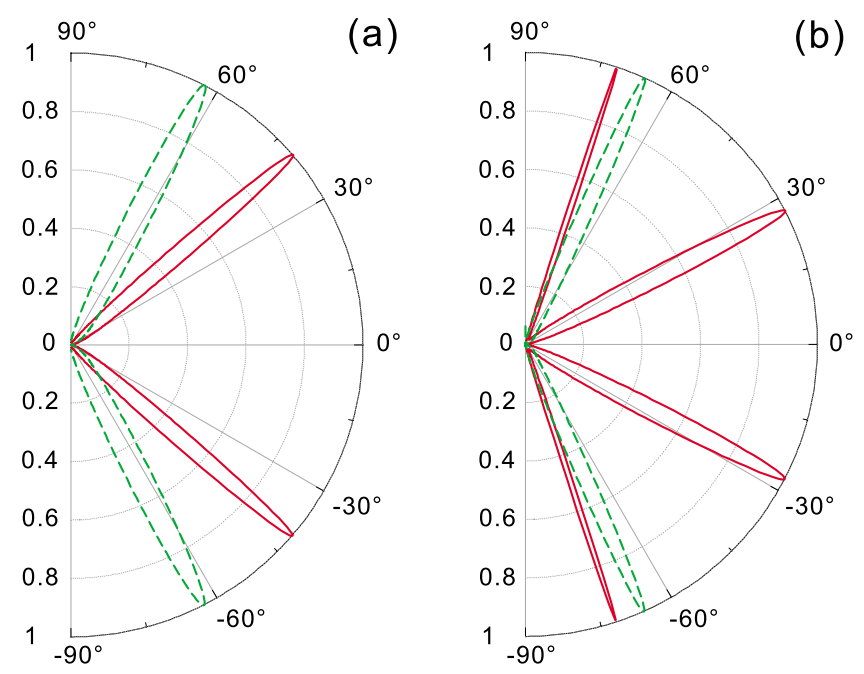

FIG. 3. (Color online) Transmission through a 100-nm-wide barrier as a function of the angle of incidence for constant energy $E=17 \mathrm{meV}$. Panel (a) results from the $4 \times 4$ Hamiltonian of Eq. (1) and panel (b) from the $2 \times 2$ one of Eq. (3). The solid red and dashed green curves are for a single barrier with height 50 and 100 $\mathrm{meV}$, respectively.

\section{B. Conductance}

It is interesting to see to what extent the transmission affects the conductance $G$, which is given by

$$
G=G_{0} \int_{-\pi / 2}^{\pi / 2} T(E, \phi) \cos \phi d \phi
$$

Here $G_{0}=2 e^{2} \sqrt{E_{F}^{2}+t_{\perp} E_{F}} L_{y} /\left(\pi h \hbar v_{F}\right), \phi$ is the angle of incidence measured from the $x$ axis, $T(E, \phi)$ is the transmission through the structure at energy $E$, and $L_{y}$ is the length of the structure along the $y$ direction.

In Fig. 4(a) we plot the conductance $G$ through 2, 5, and 10 barriers in blue, red, and black color, respectively. The height of the barriers is $50 \mathrm{meV}$, their width is $D=10 \mathrm{~nm}$, and the interbarrier distance is $L=5 \mathrm{~nm}$. The solid curves are obtained using Eq. (1) and the dashed ones using the reduced Hamiltonian of Eq. (3) with the same coupling strength $t_{\perp}$ $=390 \mathrm{meV}$. As can be seen, both models give qualitatively the same results. The disagreement is mostly apparent in the low-energy region and is mainly due to the large deviation $E-V$ of the energy from the barrier potential $V$, due to which the $2 \times 2$ Hamiltonian approximation inside the barrier fails.

The main conductance feature, for $N=2$, of panel (a) is reflected in the contour plot of the transmission shown in Fig. 4(b) with energy and angle of incidence the two axes. As can be seen, the transmission shows the same behavior as the conductance in the same energy range. Notice also that in agreement with Ref. 3, the transmission vanishes for $\phi=0$. As for the dependence of the conductance on the interbarrier distance $L$, we show it, for $N=2$, in Fig. 4 (c) for the $4 \times 4$ Hamiltonian (solid curves) and the $2 \times 2$ one (dashed 

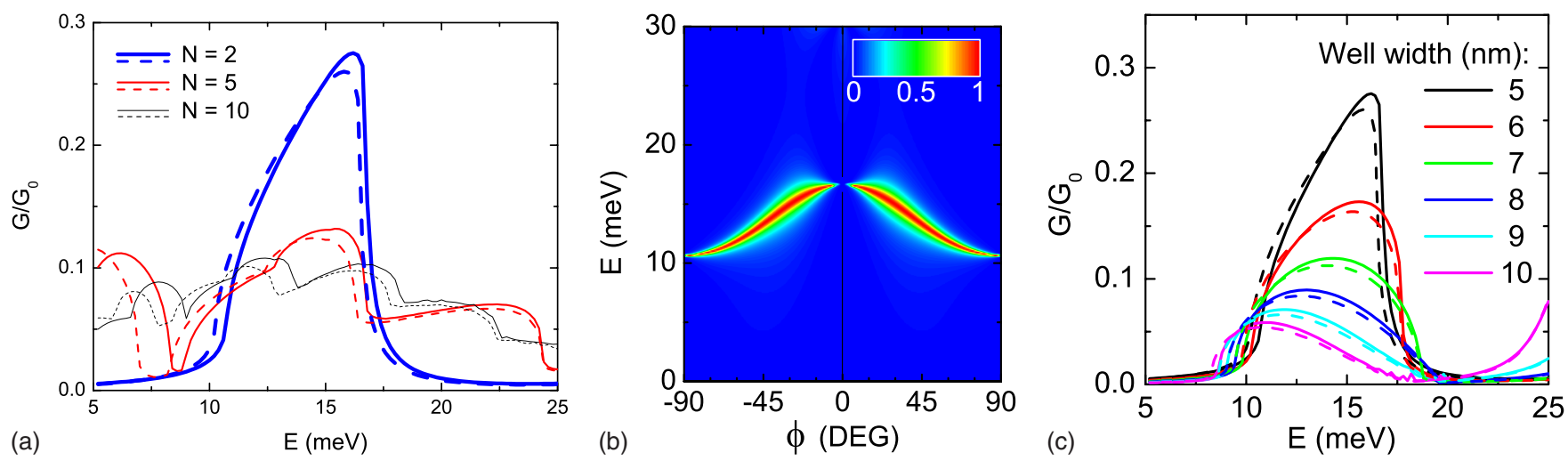

FIG. 4. (Color online) (a) Conductance as a function of energy. The very thick blue, thick red, and thin black solid curves are for 2, 5, and 10 barriers, respectively, of width $10 \mathrm{~nm}$, height $50 \mathrm{meV}$, and interbarrier distance of $5 \mathrm{~nm}$. They result from the $4 \times 4 \mathrm{Hamiltonian}$, Eq. (1), while the dashed curves result from the $2 \times 2$ one, Eq. (3). (b) Contour plot of the transmission versus energy and angle of incidence. (c) The main conductance feature of panel (a), for $N=2$, versus energy for different well widths. The solid curves are for the $4 \times 4$ Hamiltonian and the dashed ones for the $2 \times 2$ one.

curves). The results of the two models are similar; the conductance weakens and shifts to lower energies upon increasing $L$, indicating a shift of the relevant state in the well with respect to the hole states in the barriers that are responsible for it.

\section{SUPERLATTICE}

\section{A. Dispersion relation}

The model we used for a SL in graphene is shown schematically in Fig. 5. The electronic spectrum resulting from

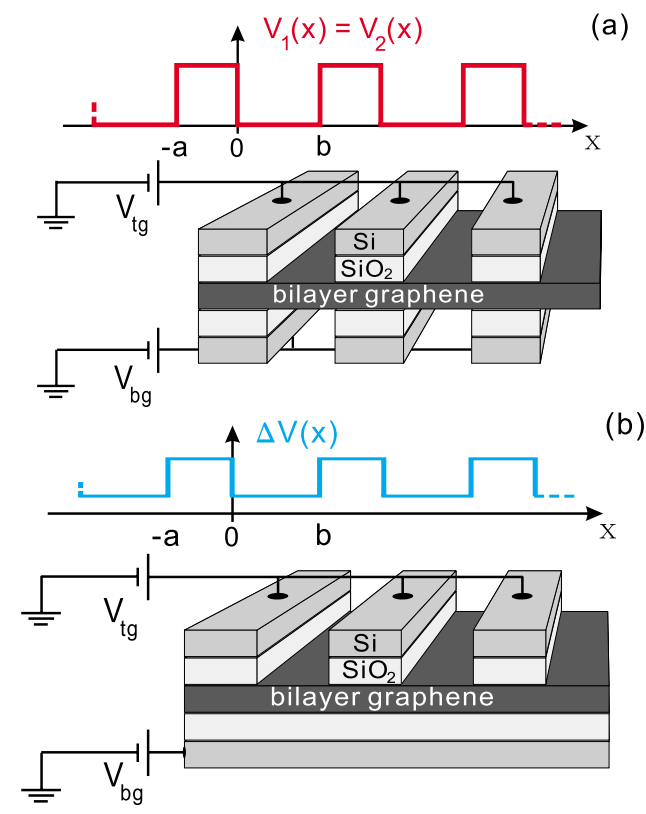

FIG. 5. (Color online) Schematics of two experimental setups for realizing the three SL potentials we investigated. In panel (a) the layer potentials $V_{1}$ and $V_{2}$ are kept the same: the experimental setup shown can be used. The setup in panel (b) can establish a bias $\Delta$ $=V_{1}-V_{2}$. In both experimental setups the layer potentials are controlled by the applied top $V_{t g}$ and back $V_{b g}$ gates. this periodic structure can be obtained by writing the solution for the spinors as Bloch waves and applying the continuity condition for the wave function at the potential steps.

In both barriers and wells the solutions are the ones for a constant potential and the boundary conditions determine the matrix relation between the wave-function coefficients in the two regions. For a periodic potential, Bloch's theorem applies with period $l=a+b$, implying $\psi_{k}(x+l)=\psi_{k}(x) e^{i k l}$. Then referring to Fig. 5 we obtain $\psi(0-)=\psi(0+)$ and $\psi(-a)$ $=\psi(b) e^{-i k l}$. Writing the wave function in the regions of constant potential as a matrix product $\Psi=\mathcal{G} \mathcal{M} \mathcal{A}$, labeling the coefficient matrices inside the barrier regions as $\mathcal{A}_{1}$ and the ones inside the well regions as $\mathcal{A}_{2}$, and applying the above boundary conditions, we obtain the matrix equations

$$
\begin{gathered}
\mathcal{G}_{1} \mathcal{A}_{1}=\mathcal{G}_{2} \mathcal{A}_{2}, \\
\mathcal{G}_{1} \mathcal{M}_{1}(-a) \mathcal{A}_{1}=\mathcal{G}_{2} \mathcal{M}_{2}(b) e^{-i k l} \mathcal{A}_{2} .
\end{gathered}
$$

Eliminating $\mathcal{A}_{1}$ in Eqs. (10) and (11) leads to

$$
\left[\mathcal{M}_{1}(-a) \mathcal{G}_{1}^{-1} \mathcal{G}_{2}-\mathcal{G}_{1}^{-1} \mathcal{G}_{2} \mathcal{M}_{2}(b) e^{-i k l}\right] \mathcal{A}_{2}=0
$$

Equating the determinant of Eq. (16) to zero,

$$
\operatorname{det}\left[\mathcal{M}_{1}(-a) \mathcal{G}_{1}^{-1} \mathcal{G}_{2}-\mathcal{G}_{1}^{-1} \mathcal{G}_{2} \mathcal{M}_{2}(b) e^{-i k l}\right]=0
$$

The solution of Eq. (14) gives the energy spectrum or dispersion relation. From this determinant we search for the zeros of Eq. (14) using the Newton method and obtain the dispersion relation.

In Fig. 6 we plot the dispersion relation versus $k_{x}$ and $k_{y}$ for three different SLs. In the first one we take the potential on the back and front gates to be the same $V=50 \mathrm{meV}$; between the strips the potential is $-50 \mathrm{meV}$. In the other two we only vary the bias difference $\Delta$ between the two layers: $\Delta=50 \mathrm{meV}$ in the barriers and $0 \mathrm{meV}$ in the wells for the second SL, and correspondingly $\Delta=50$ and $25 \mathrm{meV}$ for the 
(1)
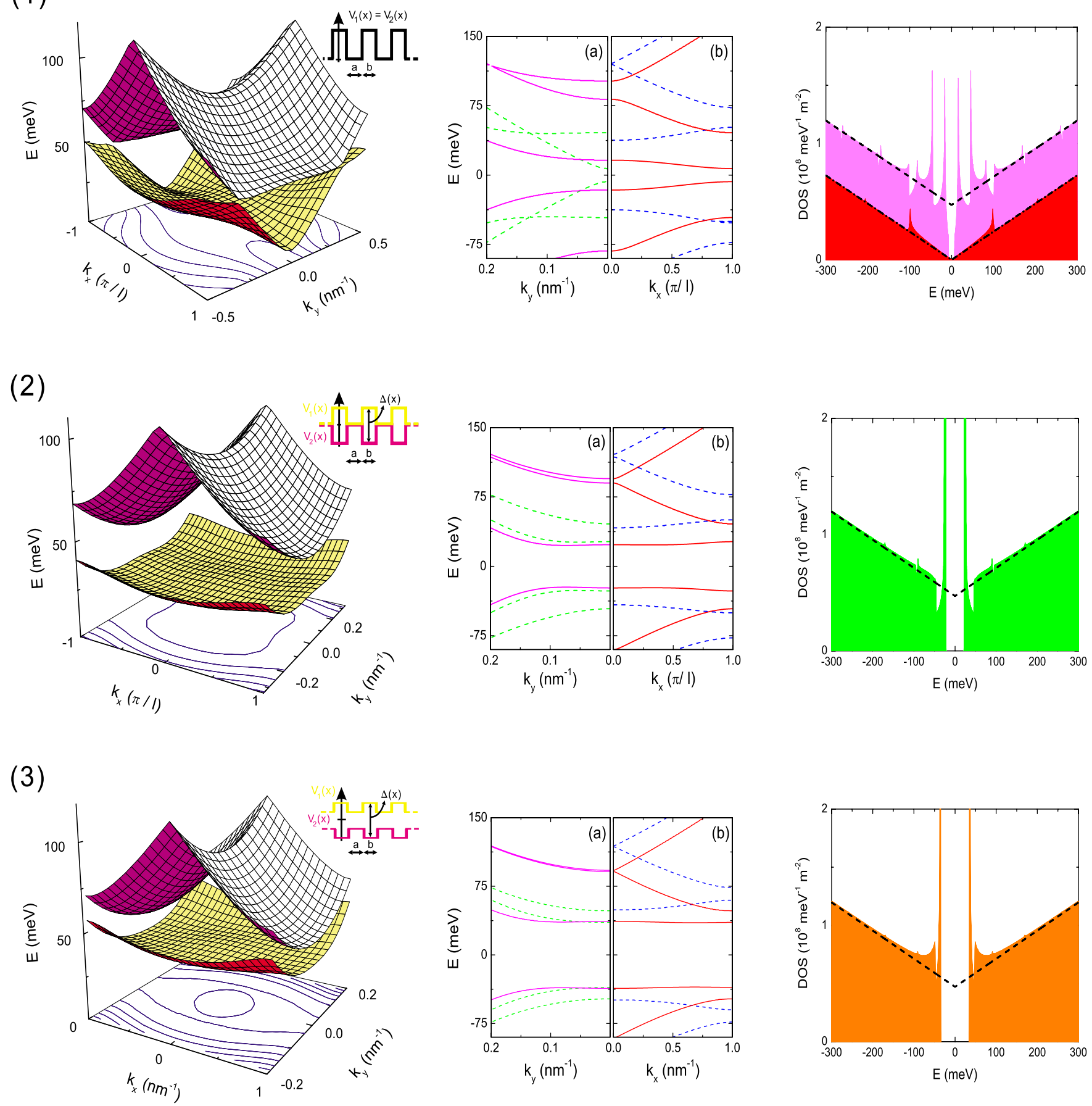

FIG. 6. (Color online) Dispersion relation and DOS for three types of SLs. (1) The barriers are $50 \mathrm{meV}$ high and the wells -50 meV deep. (2) The barriers are biased by $\Delta=50 \mathrm{meV}$, and the wells are unbiased, i.e., $\Delta=0 \mathrm{meV}$. (3) The barriers are biased by $\Delta=50 \mathrm{meV}$ and the wells by $\Delta=25 \mathrm{meV}$. Left column: energy vs $k_{x}$ and $k_{y}$ for $a=b=10 \mathrm{~nm}$ and $t_{\perp}=390 \mathrm{meV}$. Lines of constant energy, belonging to the lower miniband, are projected onto the $\left(k_{x}, k_{y}\right)$ plane. Middle column: slices of the corresponding dispersion relation, (a) for constant $k_{x}$ $=0$ (solid magenta curves) and $k_{x}=\pi / l$ (dashed green curves), and (b) for constant $k_{y}=0$ (solid red curves) and $k_{y}=0.2 / \mathrm{nm}$ (dashed blue curves). Only half the Brillouin zone is shown. Right column: DOS for the corresponding SL. For the unbiased SL (1) we also show the DOS (red area) for a SL with the same parameters on a single-layer graphene. The dashed (dashed-dotted) curves show the bilayer (single-layer) DOS in the absence of the SL potential.

third one. The average potential of both layers is kept constant. The parameters used are $a=b=10 \mathrm{~nm}$ and the tunnel coupling ${ }^{7}$ is $t_{\perp}=390 \mathrm{meV}$. Only the first two minibands are shown in the left panels of Fig. 6. The middle column shows cross sections of the dispersion relation for constant $k_{x}$ in panel (a) and constant $k_{y}$ in panel (b).

The SL potential creates minibands. For the first type of SL one can easily find an analytical expression for the one- 
dimensional case, i.e., for $k_{y}=0$. The dispersion calculated from the four-band Hamiltonian is

$$
\cos (k L)=\cos \left(\alpha_{1} a\right) \cos \left(\alpha_{2} b\right)-Q \sin \left(\alpha_{1} a\right) \sin \left(\alpha_{2} b\right),
$$

where $\varepsilon_{j}=E-V_{j}$ in region $j$ and $Q$ is given by Eq. (11). In these formulas the values of the momenta along the $x$ direction in the different regions are denoted as $\alpha_{j}=\alpha_{ \pm, j}$ $=\left[\varepsilon^{2} \pm \varepsilon_{j} t_{\perp}\right]^{1 / 2}$, both signs contribute to the dispersion relation, and the sum of the corresponding results gives the total dispersion relation. The analog of this formula for the twoband approximation of the Hamiltonian is the same formula with $\alpha_{ \pm, j}=\left[ \pm \varepsilon_{j} t_{\perp}\right]^{1 / 2}$ instead of $\alpha_{ \pm, j}=\left[\varepsilon^{2} \pm \varepsilon_{j} t_{\perp}\right]^{1 / 2}$.

In contrast to the gapless spectrum of SLs on single-layer graphene, here a band gap is found for $k_{y}=0$. This is in agreement with the fact that for the transmission through a barrier, there is no perfect transmission for perpendicular incidence while in single-layer graphene there is. The second SL potential has the same barrier and well parameters as Fig. 2(b); the resonance at $k_{x} \simeq 0.16 \mathrm{~nm}^{-1}$ which we saw in this double-barrier system seems to correspond to the energy value of the first band, $E \simeq 25 \mathrm{meV}$. Also, for the lowest band the mexican-hat energy profile of biased bilayer graphene is retained in the $k_{y}$ direction. In the third SL potential the gaps between the subbands are smaller than those of the second SL, and the dispersion relation resembles more the (folded) one of bilayer graphene without any SL potential but with an applied constant potential difference. The DOS of the latter two SLs shows large van Hove peaks at energies corresponding to the lowest band; there the velocity is also zero and localized states form.

\section{B. Density of states}

To understand part of the behavior of carriers in a SL we evaluate the DOS $D(E)$. In the reduced-zone scheme it is given by

$$
D(E)=\frac{4 A}{\pi^{2}} \sum_{n} \int_{0}^{\pi / l} d k_{x} \int_{0}^{\infty} d k_{y} \delta\left[E-E_{n}\left(k_{x}, k_{y}\right)\right],
$$

where $A$ is the surface area. The integral is evaluated numerically by converting it to a sum in the manner

$$
\int_{0}^{\pi / l} d k_{x} \int_{0}^{\infty} d k_{y} \approx\left(\frac{\pi}{N_{x} l}\right)\left(\frac{k_{\max }}{N_{y}}\right) \sum_{k_{x}=0}^{\pi / l} \sum_{k_{y}=0}^{k_{\max }},
$$

where the $k_{x}$ and $k_{y}$ indices take the values

$$
k_{x}=\frac{n_{x}}{N_{x}} \frac{\pi}{l}, \quad k_{y}=\frac{n_{y}}{N_{y}} k_{\max }, \quad n_{x}\left(n_{y}\right)=1 \cdots N_{x}\left(N_{y}\right) .
$$

The cutoff $k_{\max }$ for $k_{y}$ is chosen sufficiently large, we took $k_{\max }=2 \mathrm{~nm}^{-1}$. In addition, we replace the $\delta$ function in Eq. (15) by a Gaussian,

$$
\delta\left[E-E_{n}\left(k_{x}, k_{y}\right)\right] \approx(1 / \sqrt{2 \pi}) e^{-\left[E-E_{n}\left(k_{x}, k_{y}\right)\right]^{2} / 2 \sigma^{2}},
$$

and choose $\sigma$ to be small but sufficiently large to compensate for the discretization of $k_{x}$ and $k_{y}$, i.e., we took $\sigma$
$=0.03 \mathrm{meV}$. The evaluated DOS is shown in the right panel of Fig. 6. In these figures the magenta, green, and orange areas are for bilayer SLs and the red one is for a single-layer SL. The dashed and dashed-dotted curves show the DOS for single-layer $\left(D_{s}\right)$ and bilayer $\left(D_{b}\right)$ graphene in the absence of the SL potential given by

$$
\begin{gathered}
D_{s}(E)=|E| / h v_{F}, \\
D_{b}(E)=\left(|E|+t_{\perp} / 2\right) / h v_{F},
\end{gathered}
$$

where we used the usual tight-binding Hamiltonian ${ }^{16}$ for single-layer graphene and the one given by Eq. (1) for bilayer graphene. The peaks in the DOS have the typical $1 / \sqrt{E-E_{0}}$ behavior of $1 \mathrm{D}$ subbands.

\section{SUMMARY AND CONCLUDING REMARKS}

We evaluated the electronic transmission and conductance through a finite number of bilayer graphene barriers. Further, we obtained the dispersion relation and the DOS for a periodically biased bilayer, i.e., a bilayer in the presence of a SL potential. With the rapid progress in the field we expect that such a periodic biasing will soon be realized experimentally. Since the elastic mean-free path of carriers in high-mobility graphene layers can be of the order of hundreds of nanometers, a ballistic behavior can be expected to be observable on the length scale of the periodic structures discussed here.

For some transmission and conductance results we used both the four-band Hamiltonian given by Eq. (1) as well as the reduced two-band Hamiltonian given by Eq. (3), cf. Figs. 4 and 5. We consider the former results as more accurate than the latter ones, since the graphene bilayer spectrum obtained from the four-band Hamiltonian is known to give a better agreement with both experimental data and theoretical tight-binding calculations. ${ }^{7}$

For zero bias the dispersion relation shows a finite gap for carriers with zero momentum in the direction parallel to the barriers in contrast to the well-known results ${ }^{1,16}$ for singlelayer graphene, cf. Fig. 6. A gap also appears for a finite bias, cf. Fig. 6. We also contrasted the DOS for bilayer graphene with the corresponding one for single-layer graphene, cf. Fig. 6. We expect that all these results will be tested experimentally in the near future.

\section{ACKNOWLEDGMENTS}

This work was supported by IMEC, the Flemish Science Foundation (FWO-Vl), the Belgian Science Policy (IAP), the Brazilian Council for Research (CNPq), and the Canadian NSERC Grant No. OGP0121756.

\section{APPENDIX}

We assume solutions of the form $\Psi_{C}(x, y)=\phi_{C}(x) e^{i k_{y} y}$, where $C=A, B$. Then Eq. (1) and the Schrödinger equation $\mathcal{H} \psi=E \psi$ lead to the following equations:

$$
-i\left(\partial_{x}-k_{y}\right) \phi_{B}=\left(\varepsilon^{\prime}-\delta\right) \phi_{A}-t^{\prime} \phi_{B^{\prime}},
$$




$$
\begin{gathered}
-i\left(\partial_{x}+k_{y}\right) \phi_{A}=\left(\varepsilon^{\prime}-\delta\right) \phi_{B}, \\
-i\left(\partial_{x}+k_{y}\right) \phi_{A^{\prime}}=\left(\varepsilon^{\prime}+\delta\right) \phi_{B^{\prime}}-t^{\prime} \phi_{A}, \\
-i\left(\partial_{x}-k_{y}\right) \phi_{B^{\prime}}=\left(\varepsilon^{\prime}+\delta\right) \phi_{A^{\prime}},
\end{gathered}
$$

where $\varepsilon^{\prime} \mp \delta=\varepsilon-\left(u_{0} \pm \delta\right), \quad u_{1}=u_{0}+\delta$, and $u_{2}=u_{0}-\delta$. We solve Eq. (A1b) for $\phi_{B}$ and Eq. (A1d) for $\phi_{A^{\prime}}$ and substitute the results in Eqs. (A1a) and (A1c). This gives

$$
\begin{aligned}
& \left(\partial_{x}^{2}-k_{y}^{2}\right) \phi_{A}=-\left(\varepsilon^{\prime}-\delta\right)^{2} \phi_{A}+t^{\prime}\left(\varepsilon^{\prime}-\delta\right) \phi_{B^{\prime}}, \\
& \left(\partial_{x}^{2}-k_{y}^{2}\right) \phi_{B^{\prime}}=-\left(\varepsilon^{\prime}+\delta\right)^{2} \phi_{B^{\prime}}+t^{\prime}\left(\varepsilon^{\prime}+\delta\right) \phi_{A} .
\end{aligned}
$$

For the system of Eqs. (A1a)-(A1d) and for constant potentials the spectrum is determined by the equation

$$
\left[-k^{2}+\left(\varepsilon^{\prime}-\delta\right)^{2}\right]\left[-k^{2}+\left(\varepsilon^{\prime}+\delta\right)^{2}\right]-t^{\prime 2}\left(\varepsilon^{\prime 2}-\delta^{2}\right)=0 .
$$

Solving it leads to four bands $\left(\epsilon_{k t^{\prime}}^{2}=k^{2}+\delta^{2}+t^{\prime 2} / 2\right)$,

$$
\begin{aligned}
& \varepsilon_{ \pm}^{\prime+}=\left[\epsilon_{k t^{\prime}}^{2} \pm t^{\prime} \sqrt{4 k^{2} \delta^{2} / t^{\prime 2}+k^{2}+t^{\prime 2} / 4}\right]^{1 / 2}, \\
& \varepsilon_{ \pm}^{\prime-}=-\left[\epsilon_{k t^{\prime}}^{2} \pm t^{\prime} \sqrt{4 k^{2} \delta^{2} / t^{\prime 2}+k^{2}+t^{\prime 2} / 4}\right]^{1 / 2},
\end{aligned}
$$

and four possible wave vectors $\pm \alpha_{ \pm} \simeq k_{x}=\left(k^{2}-k_{y}^{2}\right)^{1 / 2}$,

$$
\alpha_{ \pm}=\left[\varepsilon^{\prime 2}+\delta^{2}-k_{y}^{2} \pm \sqrt{4 \varepsilon^{\prime 2} \delta^{2}+t^{\prime 2}\left(\varepsilon^{\prime 2}-\delta^{2}\right)}\right]^{1 / 2} .
$$

To obtain the general solution for the spinors we assume plane-wave solutions for $\phi_{A}=\phi_{A}^{+}+\phi_{A}^{-}$of the form

$$
\phi_{A}^{+}=A e^{i \alpha_{+} x}+B e^{-i \alpha_{+} x}, \quad \phi_{A}^{-}=C e^{i \alpha_{-} x}+D e^{-i \alpha_{-} x} .
$$

Then Eq. (A1b) gives $\left(f_{ \pm}^{ \pm}=\left[-i k_{y} \pm \alpha_{ \pm}\right] /\left[\varepsilon^{\prime}-\delta\right]\right)$

$$
\phi_{B}^{ \pm}=f_{+}^{ \pm} A e^{i \alpha_{ \pm} x}+f_{-}^{ \pm} B e^{-i \alpha_{ \pm} x},
$$

with $A, B$ replaced by $C, D$, respectively, if the lower-upper - sign is used in $\phi$ and $f$. Further, Eq. (A1a) gives

$$
\phi_{B^{\prime}}=h^{ \pm} A e^{i \alpha_{ \pm} x}+h^{ \pm} B e^{-i \alpha_{ \pm} x} \text {, }
$$

with $h^{ \pm}=\left[\left(\varepsilon^{\prime}-\delta\right)^{2}-k_{y}^{2}-\alpha_{ \pm}^{2}\right] /\left[t^{\prime}\left(\varepsilon^{\prime}-\delta\right)\right]$. Substituting $\phi_{B^{\prime}}$ in Eq. (A1d) gives

$$
\phi_{A^{\prime}}=g_{+}^{ \pm} h^{ \pm} A e^{i \alpha_{ \pm} x}+g_{-}^{ \pm} h^{ \pm} B e^{-i \alpha_{ \pm} x},
$$

where $g_{ \pm}^{ \pm}=\left[i k_{y} \pm \alpha_{ \pm}\right] /\left[\varepsilon^{\prime}+\delta\right]$; the upper \pm sign in $f$ and $g$ correspond to the subscripts of $\alpha$ and the lower ones to those in front of $\alpha$. The eigenstates are

$$
\Psi_{ \pm}^{ \pm}=N^{ \pm}\left(\begin{array}{c}
1 \\
f_{ \pm}^{ \pm} \\
h^{ \pm} \\
g_{ \pm}^{ \pm} h^{ \pm}
\end{array}\right) e^{ \pm i \alpha_{ \pm} x+i k_{y} y} .
$$

$N^{ \pm}$is a normalization constant, such that each state carries a unit current, and is given by

$$
N^{ \pm^{2}}=\frac{t^{\prime}\left(\varepsilon^{2}-\delta^{2}\right)}{2 W \alpha_{ \pm}\left[t^{\prime}\left(\varepsilon^{\prime}+\delta\right)+\left(\varepsilon^{\prime}-\delta\right)^{2}-k_{y}^{2}-\alpha_{ \pm}^{2}\right]} .
$$

The solution $\Psi=\left(\Psi_{A}, \Psi_{B}, \Psi_{B^{\prime}}, \Psi_{A^{\prime}}\right)^{T}$ can be rewritten in the matrix form

$$
\Psi=\left(\begin{array}{c}
\Psi_{A} \\
\Psi_{B} \\
\Psi_{B^{\prime}} \\
\Psi_{A^{\prime}}
\end{array}\right)=\mathcal{G M}\left(\begin{array}{c}
A \\
B \\
C \\
D
\end{array}\right)
$$

with

$$
\mathcal{G}=\left(\begin{array}{cccc}
1 & 1 & 1 & 1 \\
f_{+}^{+} & f_{-}^{+} & f_{+}^{-} & f_{-}^{-} \\
h^{+} & h^{+} & h^{-} & h^{-} \\
g_{+}^{+} h^{+} & g_{-}^{+} h^{+} & g_{+}^{-} h^{-} & g_{-}^{-} h^{-}
\end{array}\right)
$$

and

$$
\mathcal{M}=\left(\begin{array}{cccc}
e^{i \alpha_{+} x} & 0 & 0 & 0 \\
0 & e^{-i \alpha_{+} x} & 0 & 0 \\
0 & 0 & e^{i \alpha_{-} x} & 0 \\
0 & 0 & 0 & e^{-i \alpha_{-} x}
\end{array}\right) .
$$

The columns of the matrix product $\mathcal{G M}$ are the (unnormalized) eigenstates of our system. *michael.barbier@gmail.com

†takis@alcor.concordia.ca

*francois.peeters@ua.ac.be

§joaomilton.pereira@ua.ac.be

${ }^{1}$ A. H. Castro Neto, F. Guinea, N. M. R. Peres, K. S. Novoselov, and A. K. Geim, Rev. Mod. Phys. 81, 109 (2009).

${ }^{2}$ O. Klein, Z. Phys. 53, 157 (1929).

${ }^{3}$ M. I. Katsnelson, K. S. Novoselov, and A. K. Geim, Nat. Phys. 2, 620 (2006).

${ }^{4}$ B. Trauzettel, D. V. Bulaev, D. Loss, and G. Burkard, Nat. Phys. 3, 192 (2007).

${ }^{5}$ G. Giovannetti, P. A. Khomyakov, G. Brocks, P. J. Kelly, and J. van den Brink, Phys. Rev. B 76, 073103 (2007).

${ }^{6}$ B. Partoens and F. M. Peeters, Phys. Rev. B 74, 075404 (2006).

${ }^{7}$ T. Ohta, A. Bostwick, T. Seyller, K. Horn, and E. Rotenberg, Science 313, 951 (2006).

${ }^{8}$ Eduardo V. Castro, K. S. Novoselov, S. V. Morozov, N. M. R. Peres, J. M. B. Lopes dos Santos, Johan Nilsson, F. Guinea, A. K. Geim, and A. H. Castro Neto, Phys. Rev. Lett. 99, 216802 (2007).

${ }^{9}$ E. McCann and V. I. Falko, Phys. Rev. Lett. 96, 086805 (2006).

${ }^{10}$ E. McCann, Phys. Rev. B 74, 161403(R) (2006).

${ }^{11}$ J. M. Pereira, Jr., P. Vasilopoulos, and F. M. Peeters, Nano Lett. 7, 946 (2007). 
${ }^{12}$ A. Isacsson, L. M. Jonsson, J. M. Kinaret, and M. Jonson, Phys. Rev. B 77, 035423 (2008).

${ }^{13}$ J. M. Pereira, Jr., P. Vasilopoulos, and F. M. Peeters, Appl. Phys. Lett. 90, 132122 (2007).

${ }^{14}$ C. Bai and X. Zhang, Phys. Rev. B 76, 075430 (2007).
${ }^{15}$ I. Snyman and C. W. J. Beenakker, Phys. Rev. B 75, 045322 (2007).

${ }^{16}$ C.-H. Park, L. Yang, Y.-W. Son, M. L. Cohen, and S. G. Louie, Nat. Phys. 4, 213 (2008). 